\title{
Using Activated Transport in Parallel Nanowires for Energy Harvesting and Hot-Spot Cooling
}

\author{
Riccardo Bosisio, ${ }^{*}$ Cosimo Gorini, ${ }^{\dagger}$ Geneviève Fleury, and Jean-Louis Pichard \\ Service de Physique de l'État Condensé, DSM/IRAMIS/SPEC, CNRS UMR 3680, CEA Saclay, \\ 91191 Gif-sur-Yvette Cedex, France \\ (Received 25 September 2014; revised manuscript received 28 January 2015; published 8 May 2015)

\begin{abstract}
We study arrays of parallel doped semiconductor nanowires in a temperature range where the electrons propagate through the nanowires by phonon-assisted hops between localized states. By solving the random-resistor-network problem, we compute the thermopower $S$, the electrical conductance $G$, and the electronic thermal conductance $K^{e}$ of the device. We investigate how those quantities depend on the position - which can be tuned with a back gate - of the nanowire impurity band with respect to the equilibrium electrochemical potential. We show that large power factors can be reached near the band edges, when $S$ self-averages to large values while $G$ is small but scales with the number of wires. Calculating the amount of heat exchanged locally between the electrons inside the nanowires and the phonons of the environment, we show that phonons are mainly absorbed near one electrode and emitted near the other when a charge current is driven through the nanowires near their band edges. This phenomenon could be exploited for a field control of the heat exchange between the phonons and the electrons at submicron scales in electronic circuits. It could be also used for cooling hot spots.
\end{abstract}

DOI: 10.1103/PhysRevApplied.3.054002

\section{INTRODUCTION}

A good thermoelectric machine must be efficient at converting heat into electricity and also must provide a substantial electric-output power for practical applications. In the linear response regime, this requires optimizing simultaneously the figure of merit $Z T=S^{2} G T /\left(K^{e}+K^{\mathrm{ph}}\right)$ and the power factor $\mathcal{Q}=S^{2} G, T$ being the operating temperature, $S$ the device thermopower, $G$ its electrical conductance, and $K^{e}$ and $K^{\text {ph }}$ its electronic and phononic thermal conductances, respectively. In the quest for highperformance thermoelectrics, semiconductor nanowires (NWs) play a front role [1-6], apparently offering the best of three worlds: first, an enhanced $S$ due to strongly broken and gate-tunable particle-hole symmetry [4,7-9], second, a suppressed $K^{\mathrm{ph}}$ by virtue of reduced dimensionality [2,3], and, finally, a high power output thanks to scalability, i.e., parallel stacking [2,5,10-17].

The perspective of developing competitive thermoelectric devices with the standard building blocks of the semiconductor industry has raised great interest in the scientific community over the past decade. On a technological standpoint, much effort has been put into the synthesis of dense NW arrays with controlled NW diameter, length, doping, and crystal orientation [11-14,16-18]. Arrays made out of various semiconductor materials including, e.g., silicon, silicon germanium, indium

\footnotetext{
*Present address: NEST, Istituto Nanoscienze, Piazza San Silvestro 12, 56127 Pisa, Italy.

${ }^{\dagger}$ Present address: Institut für Theoretische Physik, Universität Regensburg, 93040 Regensburg, Germany.
}

arsenide, or bismuth telluride have thus been investigated. Versatile measurement platforms have been developed to access the set of thermoelectric coefficients, and the feasibility of NW-based thermoelectric modules has been assessed $[2,5,10,15,19,20]$. On the theoretical side, numerous calculations of $S, G, K^{e}$, and $K^{\mathrm{ph}}$ of various single NWs have been carried out in the ballistic regime of electronic transport [21-26] or in the diffusive regime [22] where a semiclassical Boltzmann approach can be used [27-38]. In two recent works $[39,40]$, we took a different approach by considering the presence of electronic localized states randomly distributed along the NWs and making up an impurity band in the semiconductor band gap. Such states are known to play a leading role in thin nanowires, when localization effects are enhanced by low dimensionality and the system size rapidly exceeds the electron localization length. After a first study devoted to the low-temperature coherent regime [39], we investigated the phonon-assisted hopping regime [40] taking place at higher temperatures and usually referred to as the Mott activated regime. For a long time, thermoelectric transport in this regime has been somewhat overlooked in the theoretical literature (with the exception of a few older works on bulk semiconductors [41-44]). In fact, the problem of thermally activated thermoelectric transport in NWs has been revisited only recently by Jiang, Entin-Wohlman, and Imry in Refs. $[45,46]$. However, the case of gated NWs where band edges are approached has not been considered, though band-edge transport, where particle-hole asymmetry is maximal, is acknowledged to be the critical one for thermoelectric conversion [47,48]. In our previous paper [40], we studied the behavior of the thermopower $S$ and of 
the electrical conductance $G$ of single disordered and gated NWs in the activated regime. We obtained near the band edges a substantial enhancement of the typical thermopower $S_{0}$ but also, unsurprisingly, a decrease of the typical conductance $G_{0}$ and large sample-to-sample fluctuations of both $G$ and $S$. This is unsatisfactory if a reliable and efficient thermoelectric device is to be realized.

In the present paper, we circumvent the latter shortcomings by considering a large set of NWs stacked in parallel in the field-effect transistor (FET) device configuration. Besides assessing the opportunities offered by bandedge-activated transport for energy harvesting, we show that activated transport through such a device can be used for an electrostatic control of the heat exchange between the phonons and the electrons at submicron scales: Injecting the carriers through the NWs gives rise to a local cooling (heating) effect near the source (drain) electrode when the chemical potential of the device probes the lower NW band edge (and conversely when it probes the upper edge). This opens promising perspectives for a local management of heat and for cooling hot spots in microelectronics.

Hereafter, we study arrays of doped semiconductor NWs, arranged in parallel and attached to two electrodes. The NWs can be either suspended or deposited onto an electrically and thermally insulating substrate. A metallic gate beneath the sample is used to vary the carrier density inside the NWs. This corresponds to a setup in the FET configuration, as sketched in Fig. 1. If the thermopower or the thermal conductances are to be investigated, a heater (not shown in Fig. 1) is added on one side of the sample to induce a temperature gradient between the electrodes. We focus on a temperature range where the activated regime proposed by Mott [variable-range hopping (VRH) regime] takes place, assuming (i) that phonon-assisted transport occurs between localized states of the NW impurity band only and (ii) that the substrate, or the NWs themselves if they are suspended, acts as a phonon bath to which NW
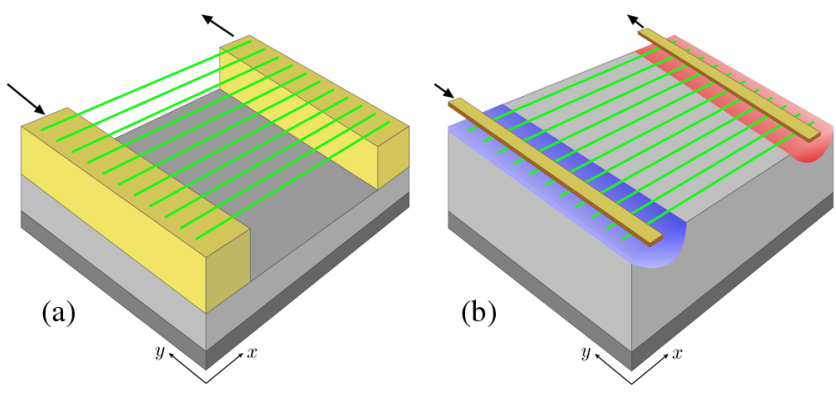

FIG. 1. Array of suspended (a) and deposited (b) parallel NWs in the FET configuration. The NWs are drawn in green, the two metallic electrodes in yellow, the substrate in gray, and the back gate in dark gray. The blue (red) spot in (b) indicates the substrate region that is cooled down (heated up) in the phonon-assisted activated regime, when a charge current flows from the left to the right electrode and the gate voltage is tuned so as to probe the lower edge of the NW impurity band. charge carriers are well coupled. We thus consider intermediate temperatures, where the thermal energy $k_{B} T$ is high enough to allow inelastic hopping between Anderson localized states of different energies (typically a few kelvins) yet low enough to keep localization effects. Such a VRH regime is observed up to room temperatures in threedimensional amorphous semiconductors [49] and very likely up to higher temperatures in the one-dimensional limit. Following Refs. $[40,45,46]$, we solve numerically the Miller-Abrahams random-resistor-network problem [50] for obtaining $S, G$, and $K^{e}$. This allows us to identify also the regions where heat exchanges between the electrons and the phonons dominantly take place in the activated regime, notably when the chemical potential probes the edges of the NW impurity band.

The model used throughout the paper is presented in Sec. II, together with a summary of the method. We find in Sec. III that, once a large set of NWs is stacked in parallel, the strong $G, S$, and $K^{e}$ fluctuations are suppressed. Denoting by $G_{0}, S_{0}$, and $K_{0}^{e}$ the typical values for a single $\mathrm{NW}$, we observe more precisely that the thermopower of a large NW array self-averages $\left(S \rightarrow S_{0}\right)$ while its electrical and electronic thermal conductances $G \rightarrow M G_{0}$ and $K^{e} \rightarrow$ $M K_{0}^{e}$ as the number $M$ of wires in parallel increases (see Fig. 2). Taking full advantage of the gate, we move close to the impurity band edges, where we recently obtained a drastic $S_{0}$ enhancement [40]. We show in Sec. IV that in this regime a large $S_{0}$ partly compensates an exponentially small $G_{0}$, so that substantial values of the power factor $\mathcal{Q} \approx M S_{0}^{2} G_{0}$ can be reached upon stacking plenty of NWs in parallel [see Fig. 3(a)]. Remarkably, the electronic figure of merit $Z_{e} T=S^{2} G T / K^{e}$ is also found to reach promising values $Z_{e} T \approx 3$ when $\mathcal{Q}$ is maximal [see Fig. 3(b)]. Furthermore, we discuss how the phononic thermal conductance $K^{\mathrm{ph}}$ will inevitably reduce the full figure of merit $Z T$ and argue that, even if record high $Z T$ is probably not to be sought in such setups, the latter have the great advantage of offering at once high output power and reasonable efficiency with standard nanotechnology building blocks.

The most important result of this paper is given in Sec. V. We study how deposited NWs in the FET configuration can be used to manage heat in the substrate, generating hot or cold spots "on demand." The idea is simple to grasp and relies on the calculation of the local heat exchanges between the NW electrons and the substrate phonons: When the gate voltage is adjusted such that the equilibrium electrochemical potential $\mu$ (defined in the electronic reservoirs) roughly coincides with one (say, the lower) impurity band edge, basically all energy states in the NWs lie above $\mu$. Therefore, if charge carriers injected into the system around $\mu$ are to gain the other end, they need to (on the average) absorb phonons at the entrance so as to jump to available states and then to release phonons when tunneling out (again at $\mu$ ). This generates in the nearby substrate regions cold strips near the injecting electrode and hot strips 
near the drain electrode [see Figs. 1(b) and 4]. These strips get scrambled along the nanowires if $\mu$ does not probe the edges of the NW impurity band. Such reliable and tunable cold spots may be exploited in devising thermal management tools for high-density circuitry, wherever increasing power densities have become a critical issue [51]. Moreover, the creation (annihilation) of the cold (hot) strips can be controlled by the back-gate voltage. Note that the underlying mechanism governing the physics of VRH transport at the NW band edges is somewhat reminiscent of the mechanism of "cooling by heating" put forward in Refs. [52-56], which also exploits the presence of a third bosonic bath in addition to the two electronic reservoirs. In our case, bosons are phonons provided mainly by the substrate; in other setups, bosons are photons provided by laser illumination (or more simply by the Sun for a photovoltaic cell). All those studies fall into the growing category of works dealing with boson-assisted electronic transport that have been shown to open promising perspectives for heat management.

\section{MODEL AND METHOD}

Architecture and/or material-specific predictions, though very important for practical engineering purposes, are however not our concern at present. On the contrary, our goal is to reach conclusions which are as general as possible, relying on a bare-bone but widely applicable Anderson model devised to capture the essentials of the physics we are interested in. We consider a set of $M$ NWs in parallel. Each NW is modeled as a chain of length $L$ described by a one-dimensional (1D) Anderson tight-binding Hamiltonian with on-site disorder [40]:

$$
\mathcal{H}=-t \sum_{i=1}^{N-1}\left(c_{i}^{\dagger} c_{i+1}+\text { H.c. }\right)+\sum_{i=1}^{N}\left(\epsilon_{i}+V_{g}\right) c_{i}^{\dagger} c_{i} .
$$

Here $N$ is the number of sites in the chain $(L=N a$ with $a$ lattice spacing), $c_{i}^{\dagger}$ and $c_{i}$ are the electron creation and annihilation operators, respectively, on site $i$, and $t$ is the hopping energy (interwire hopping is neglected). We assume that no site can be doubly occupied due to Coulomb repulsion but otherwise neglect interactions [57]. The site energies $\epsilon_{i}$ are uncorrelated random numbers uniformly distributed in the interval $[-W / 2, W / 2]$, while $V_{g}$ is a constant (tunable) potential due to the back gate. The electronic states are localized at certain positions $x_{i}$ with localization lengths $\xi_{i}$ and eigenenergies $E_{i}$. The $E_{i}$ 's lie within the NW impurity band whose center can be shifted with the gate voltage $V_{g}$. For simplicity's sake, we generate randomly the positions $x_{i}$ along the chain (with a uniform distribution) and assume $\xi_{i}=\xi\left(E_{i}\right)$, where $\xi(E)$ characterizes the exponential decay of the typical conductance $G_{0} \sim \exp (-2 L / \xi)$ of the 1D Anderson model at zero temperature and energy $E$. Analytical expressions giving $\xi(E)$ in the weak disorder limit of the Anderson model are given in Ref. [39].
The NWs are attached to two electronic reservoirs $L$ and $R$ and to a phonon bath; i.e., the system is in a three-terminal configuration. Particles and heat (energy) can be exchanged with the electrodes, but only heat (energy) with the phonon bath. At equilibrium the whole system is thermalized at a temperature $T$, and both $L$ and $R$ are at electrochemical potential $\mu$ (set to $\mu \equiv 0$, at the band center when $V_{g}=0$ ). A voltage and/or temperature bias between the electrodes drives an electron current through the NWs. Hereafter we consider the linear response regime, valid when small biases $\delta \mu \equiv \mu_{L}-\mu_{R}$ and $\delta T \equiv T_{L}-T_{R}$ are applied.

We study the inelastic activated regime. Following Ref. [46], we assume that the charge carriers (say, electrons of charge $e$ ) tunnel elastically from reservoir $\alpha=L, R$ into some localized states $i$ whose energies $E_{i}$ are located in a window of the order of $k_{B} T_{\alpha}$ around $\mu_{\alpha}$. They then proceed via phonon-assisted hops to the other end, finally tunneling out. The maximal carrier hop along the NWs is of the order of Mott length $L_{M}$ in space (or Mott energy $\Delta$ in energy) [40]. At the lowest temperatures considered in this work, $\xi(\mu) \ll L_{M} \ll L$ and transport is of the VRH type. An increasing temperature shortens $L_{M}$ until $L_{M} \approx \xi(\mu)$, when the nearest-neighbor hopping $(\mathrm{NNH})$ regime is reached. The crossover VRH $\rightarrow$ NNH takes place roughly at Mott temperature $T_{M}$, whose dependence on $V_{g}$ can be found in Ref. [40].

The total electron and heat currents flowing through the whole array are calculated by solving the randomresistor-network problem [50,57]. The method is summarized in Appendix A. It takes as input parameters the rate $\gamma_{e}$ quantifying the coupling between the NW (localized) and the reservoir (extended) states, and the rate $\gamma_{e \text {-ph }}$ measuring the coupling to the NWs and/or substrate phonons. We point out that we go beyond the usual approximation $[46,50,57]$ neglecting the $\xi_{i}$ variations from state to state $\left[\xi_{i} \approx \xi(\mu)\right.$ ], the latter being inappropriate close to the band edges, where $\xi_{i}$ varies strongly with the energy. Following Ref. [40], the random-resistor network is then solved for $\xi_{i} \neq \xi_{j}$. The particle and heat currents thus obtained are related to the small imposed biases $\delta \mu, \delta T$ via the Onsager matrix [58], which gives access to $G, K^{e}$, and $S$.

\section{SCALING OF THE THERMOELECTRIC COEFFICIENTS WITH THE NUMBER OF NANOWIRES}

The typical conductance $G_{0}$ and thermopower $S_{0}$ of a single NW are studied in Ref. [40]. They are defined as the median of the distribution of $\ln G$ and $S$, obtained when considering a large statistical ensemble of disorder configurations. In Fig. 2, we show that, if the system is made of a sufficiently large number $M$ of parallel NWs, the overall electrical conductance scales as the number of wires times the typical value $\left(G \approx M G_{0}\right)$, while the thermopower averages out to the typical value of a single wire $\left(S \approx S_{0}\right)$. For completeness, the mean values are also shown and seen to be a less accurate estimate. As expected, convergence is 

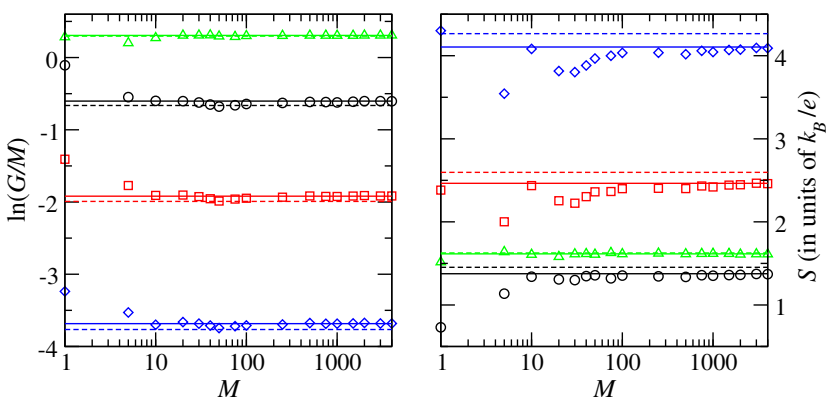

FIG. 2. Convergence of $G / M$ (left, in units of $e^{2} / \hbar$ ) and $S$ (right) with the number $M$ of parallel NWs. Symbols correspond to $V_{g}=1.9 t$ (circle), $2.1 t$ (square), and 2.3t (diamond) at $k_{B} T=0.1 t$ and $V_{g}=1.9 t$ at $k_{B} T=0.5 t$ (triangle). The horizontal lines indicate the corresponding mean values (dashed lines) and typical values (solid lines) of $\ln G$ and $S$ of a single wire $(M=1)$. Parameters: $W=t, \gamma_{e}=\gamma_{e-\mathrm{ph}}=t / \hbar$, and $L=450 a$.

faster at higher temperatures. Note that identical results have been obtained for the electronic thermal conductance $K^{e} \approx M K_{0}^{e}$ (not shown).

\section{POWER FACTOR AND FIGURE OF MERIT}

By stacking a large number $M$ of NWs in parallel, the device power factor can be enhanced $\mathcal{Q} \approx M S_{0}^{2} G_{0}$ without affecting its electronic figure of merit $Z_{e} T \approx S_{0}^{2} G_{0} T / K_{0}^{e}$. Figure 3 shows how the asymptotical $\mathcal{Q} / M$ and $Z_{e} T$ values (reached when $M \gtrsim 100$ ) depend on the gate voltage $V_{g}$ and on the temperature $T$. We observe in Fig. 3(a) that the power factor is maximum for $\mu$ close to the impurity band edge (black solid line) and for VRH temperatures. This parameter range represents the best compromise between two opposite requirements: maximizing the thermopower (hence favoring low $T$ and large $V_{g}$ ) while keeping a reasonable electrical conductance (favoring instead higher $T$ and $V_{g} \approx 0$ ). Formulas previously reported [40], giving the $T$ and $V_{g}$ dependence of $G_{0}$ and $S_{0}$, let us predict that $\mathcal{Q}$ is maximal when $\left|S_{0}\right|=2 k_{B} /|e| \approx 0.2 \mathrm{mV} \mathrm{K}^{-1}$ (black dashed line). A comparison between Figs. 3(a) and 3 (b) reveals that, in the parameter range corresponding to the best power factor $\left(V_{g} \sim 2.5 t, k_{B} T \sim 0.6 t\right), Z_{e} T \simeq 3$, a remarkably large value. Much larger values of $Z_{e} T$ could be obtained at lower temperatures or far outside the band, but they are not of interest for practical purposes since in those regions $\mathcal{Q}$ is vanishing. In Appendix $\mathrm{B}, \mathcal{Q}$ and $Z_{e} T$ are shown to be roughly independent of the NW length $L$ (for $L \gtrsim L_{M}$ ) in the temperature and gate voltage ranges explored in Fig. 3. Moreover $\mathcal{Q} / \gamma_{e}$ and $Z_{e} T$ are almost independent of the choice of the parameters $\gamma_{e}$ and $\gamma_{e-\mathrm{ph}}$, provided $\gamma_{e \text {-ph }} \gtrsim \gamma_{e}$ (see Appendix C). When $\gamma_{e \text {-ph }}<\gamma_{e}$, both quantities are found to be (slightly) reduced.

Let us now estimate the order of magnitude of the device performance. The substrate (or the NWs themselves if they are suspended) is assumed to supply enough phonons to the NW charge carriers for the condition $\gamma_{e-\mathrm{ph}} \gtrsim \gamma_{e}$ to hold.
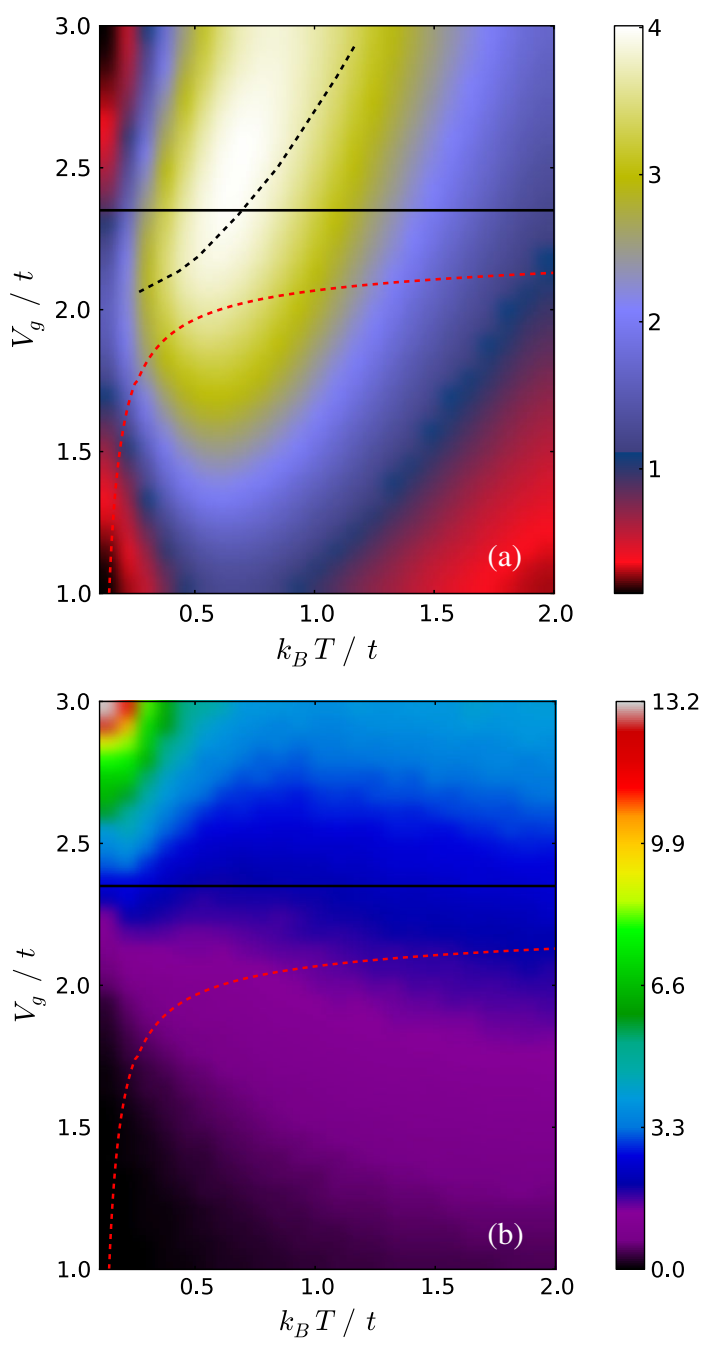

FIG. 3. $\mathcal{Q} / M$ in units of $k_{B}^{2} / \hbar$ (a) and $Z_{e} T$ (b) as a function of $T$ and $V_{g}$. Data are shown in the large $M$ limit $(M=150)$ where there is self-averaging. The horizontal lines give $V_{g}$ 's value at which the band edge is probed at $\mu$ [below (above) it, one probes the inside (outside) of the impurity band]. The red dashed lines $T=T_{M}$ separate the $\mathrm{VRH}\left(T \lesssim T_{M}\right)$ and the $\mathrm{NNH}\left(T \gtrsim T_{M}\right)$ regimes. The black dashed line in (a) is the contour along which $S_{0}=2 k_{B} / e$. Parameters: $W=t, \gamma_{e}=\gamma_{e-\mathrm{ph}}=t / \hbar$, and $L=450 a$.

Besides, we keep explicit the $\gamma_{e}$-linear dependence of $\mathcal{Q}$ (and of $K_{0}^{e}$ that will soon be needed). $\gamma_{e}$ depends on the quality of the metal-NW contact. We estimate it to be within the range $0.01-1$ in units of $t / \hbar$, where $t / k_{B} \approx 150 \mathrm{~K}$ throughout [59]. This yields $\gamma_{e} \approx(0.02-2) \times 10^{13} \mathrm{~s}^{-1}$. For the sake of brevity, we introduce the dimensionless number $\tilde{\gamma}_{e}=\gamma_{e} \hbar / t$. Focusing on the region of Fig. 3(a), where the power factor is maximal, we evaluate the typical output power and figure of merit that can be expected. We first notice that power factor $\mathcal{Q} / M \approx 4 k_{B}^{2} / h$ maximum values in Fig. 3(a), obtained with $\tilde{\gamma}_{e}=1$, would yield $\mathcal{Q} \approx 7 \tilde{\gamma}_{e} \times 10^{-7} \mathrm{~W} \mathrm{~K}^{-2}$ for a chip with $M \approx 10^{5}$ parallel NWs. Since $\mathcal{Q}$ controls the maximal output power $P_{\max }$ that can be extracted from the setup as $P_{\max }=\mathcal{Q}(\delta T)^{2} / 4$ 
[60], one expects $P_{\max } \approx 20 \tilde{\gamma}_{e} \mu \mathrm{W}$ for a small temperature bias $\delta T \approx 10 \mathrm{~K}$. In this region a large value $Z_{e} T \approx 3$ is obtained, but, to estimate the full figure of merit $Z T=Z_{e} T /\left(1+K^{\mathrm{ph}} / K^{e}\right)$, the phononic part $K^{\mathrm{ph}}$ of the thermal conductance must also be taken into account. To limit the reduction of $Z T$ by phonons, the setup configuration with suspended nanowires is preferable [Fig. 1(a)]. In this case $K^{\mathrm{ph}} \approx M K_{0}^{\mathrm{NW}}, K_{0}^{\mathrm{NW}}$ being the typical phononic thermal conductance of a single NW, and has to be compared to $K^{e} \approx M K_{0}^{e}$. By introducing the corresponding conductivities $\kappa$, the ratio $K^{\mathrm{ph}} / K^{e} \approx \kappa_{0}^{\mathrm{NW}} / \kappa_{0}^{e}$ is to be estimated. Our numerical results obtained for 1D NWs show $K_{0}^{e} \approx 1.5 \tilde{\gamma}_{e} k_{B} t / \hbar$ in the range of interest where $\mathcal{Q}$ is maximal and $Z_{e} T \approx 3$ (at $V_{g}=2.5 t$ and $k_{B} T=0.6 t$, keeping other parameters in Fig. 3 unchanged). To deduce the corresponding conductivity $\kappa_{0}^{e}$, the NW aspect ratio must be specified. We consider, for instance, the case of $1-\mu \mathrm{m}$-long NWs with a diameter of $20 \mathrm{~nm}$, for which our pure 1D model is expected to hold [61], at least semiquantitatively. Thereby we get $\kappa_{0}^{e} \approx 1 \tilde{\gamma}_{e} \mathrm{~W} /(\mathrm{Km})$, while the measured thermal conductivity of $\mathrm{Si}$ NWs of similar geometry is $\kappa_{0}^{\mathrm{NW}} \approx 2 \mathrm{~W} /(\mathrm{Km})$ at $T \approx 100 \mathrm{~K}$ [62]. We thus evaluate for suspended NWs $Z T \approx Z_{e} T /\left(1+2 / \tilde{\gamma}_{e}\right)$, i.e., $Z T \approx 0.01-1$ for $Z_{e} T \approx 3$ and $\tilde{\gamma}_{e}=0.01-1$. Those estimations, though rough, are extremely encouraging, as they show us that such a simple and Si-based device shall generate high electrical power from wasted heat (scalable with $M$, for $M$ large enough) with a fair efficiency (independent of $M$, for $M$ large enough).

Let us note that maximizing $\gamma_{e}$ is important for achieving high $\mathcal{Q}$ and $Z T$. However, at the same time $\gamma_{e \text {-ph }} \gtrsim \gamma_{e}$ should preferably hold. If the NWs themselves do not ensure a large enough $\gamma_{e-\mathrm{ph}}$, the use of a substrate providing phonons is to be envisaged. Yet, this will add a detrimental contribution $K^{\text {sub }}$ to $K^{\mathrm{ph}}$. In general, the substrate cross section $\left(\Sigma^{\text {sub }}\right)$ will be substantially larger than the NW one $\left(M \Sigma^{\mathrm{NW}}\right)$. Thus, even for a good thermal insulator such as $\mathrm{SiO}_{2}$, with thermal conductivity $\kappa^{\text {sub }} \approx 0.7 \mathrm{~W} /(\mathrm{Km})$ at $T \approx 100 \mathrm{~K}$ [63], $Z / Z_{e}=\left[1+\left(\kappa^{\mathrm{sub}} \Sigma^{\mathrm{sub}}+M \kappa_{0}^{\mathrm{NW}} \Sigma^{\mathrm{NW}}\right) / M \kappa_{0}^{e} \Sigma^{\mathrm{NW}}\right]^{-1} \ll 1$. Better ratios $Z / Z_{e}$ could be obtained for substrates with lower $K^{\text {sub }}$ (silica aerogels [64], porous silica [65], very thin substrate layer), but they will not necessarily guarantee a good value of $\gamma_{e \text {-ph }}$ (and hence of $Z_{e}$ ). Clearly, finding a balance between a large $\gamma_{e-\mathrm{ph}}$ and a low $K^{\mathrm{ph}}$ is a material engineering optimization problem. Though the presence of a substrate appears detrimental for efficiently harvesting electrical energy from the wasted heat, we shall now see how it could be used for heat management at the nanoscale.

\section{GATE-CONTROLLED CREATION OR ANNIHILATION OF COLD AND HOT STRIPS}

Hereafter, we consider the deposited setup sketched in Fig. 1(b) and assume a constant temperature $T$ everywhere.
An intriguing feature of this three-terminal setup is the possibility to generate or control hot and cold spots close to the substrate boundaries by applying a bias $\delta \mu / e$. This effect is a direct consequence of the heat-exchange mechanism between electrons in the NWs and phonons in the substrate. Indeed, given a pair of localized states $i$ and $j$ inside a NW, with energies $E_{i}$ and $E_{j}$, respectively, the heat current absorbed from (or released to) the phonon bath by an electron in the transition $i \rightarrow j$ is $I_{i j}^{Q}=\left(E_{j}-E_{i}\right) I_{i j}^{N}, I_{i j}^{N}$ being the hopping particle current between $i$ and $j$ [40]. The overall hopping heat current through each localized state $i$ is then found by summing over all but the $i$ th states:

$$
I_{i}^{Q}=\sum_{j} I_{i j}^{Q}=\sum_{j}\left(E_{j}-E_{i}\right) I_{i j}^{N}
$$

with the convention that $I_{i}^{Q}$ is positive (negative) when it enters (leaves) the NWs at site $i$. Since the energy levels $E_{i}$ are randomly distributed, the $I_{i}^{Q}$, s (and, in particular, their sign) fluctuate from site to site (see Fig. 8 in Appendix D for an illustration). The physically relevant quantities are, however, not the $I_{i}^{Q}$ 's but rather their sum within an area $\Lambda_{\mathrm{ph}} \times \Lambda_{\mathrm{ph}}$, where $\Lambda_{\mathrm{ph}}$ is the phonon thermalization length in the substrate (i.e., the length over which a local substrate temperature can be defined; see Appendix D for an estimation). Given a point $(x, y)$ and a $\Lambda_{\mathrm{ph}} \times \Lambda_{\mathrm{ph}}$ area centered around it, such a sum is denoted $\mathcal{I}_{x, y}^{Q}$. If $\mathcal{I}_{x, y}^{Q}>0$ $(<0)$, a volume $\Lambda_{\mathrm{ph}}^{3}$ of the substrate beneath $(x, y)$ is cooled (heated) [66]. Deeper than $\Lambda_{\mathrm{ph}}$ away from the surface, the equilibrium temperature $T$ is reached.

Figure 4 shows how $\mathcal{I}_{x, y}^{Q}$ depends on the coordinates $x, y$ in the two-dimensional parallel NW array. The left and right panels show, respectively, the situation in the absence of a gate voltage, when charge carriers tunnel into or out of NWs at the impurity band center, and the opposite situation when a large gate voltage is applied in order to inject or extract carriers at the band bottom. In both cases, two values of the temperature are considered [top (bottom) panels]. All other parameters are fixed. Note that data are plotted for the model introduced in Sec. II, having estimated $a \approx 3.2 \mathrm{~nm}, t / k_{B} \approx 150 \mathrm{~K}$, and $\Lambda_{\mathrm{ph}} \approx$ $480[240] \mathrm{nm} \approx 150[75] a$ for $\mathrm{S} \mathrm{SiO}_{2}$ substrate at the temperatures considered, $T=0.25[0.5] t / k_{B} \approx 37.5[75] \mathrm{K}$. Those estimates are discussed in Appendix D. In the left panels of Fig. 4, the heat maps show puddles of positive and negative $\mathcal{I}_{x, y}^{Q}$, corresponding, respectively, to cooled and heated regions in the substrate below the NW array. They are the signature of random absorption and emission of substrate phonons by the charge carriers, all along their propagation through the NWs around the band center. In the right panels, the regions of positive and negative $\mathcal{I}_{x, y}^{Q}$ are, respectively, confined to the NW entrance and exit. This is due to the fact that charge carriers entering the NWs at $\mu$ around the band bottom find available states to jump to (at a 

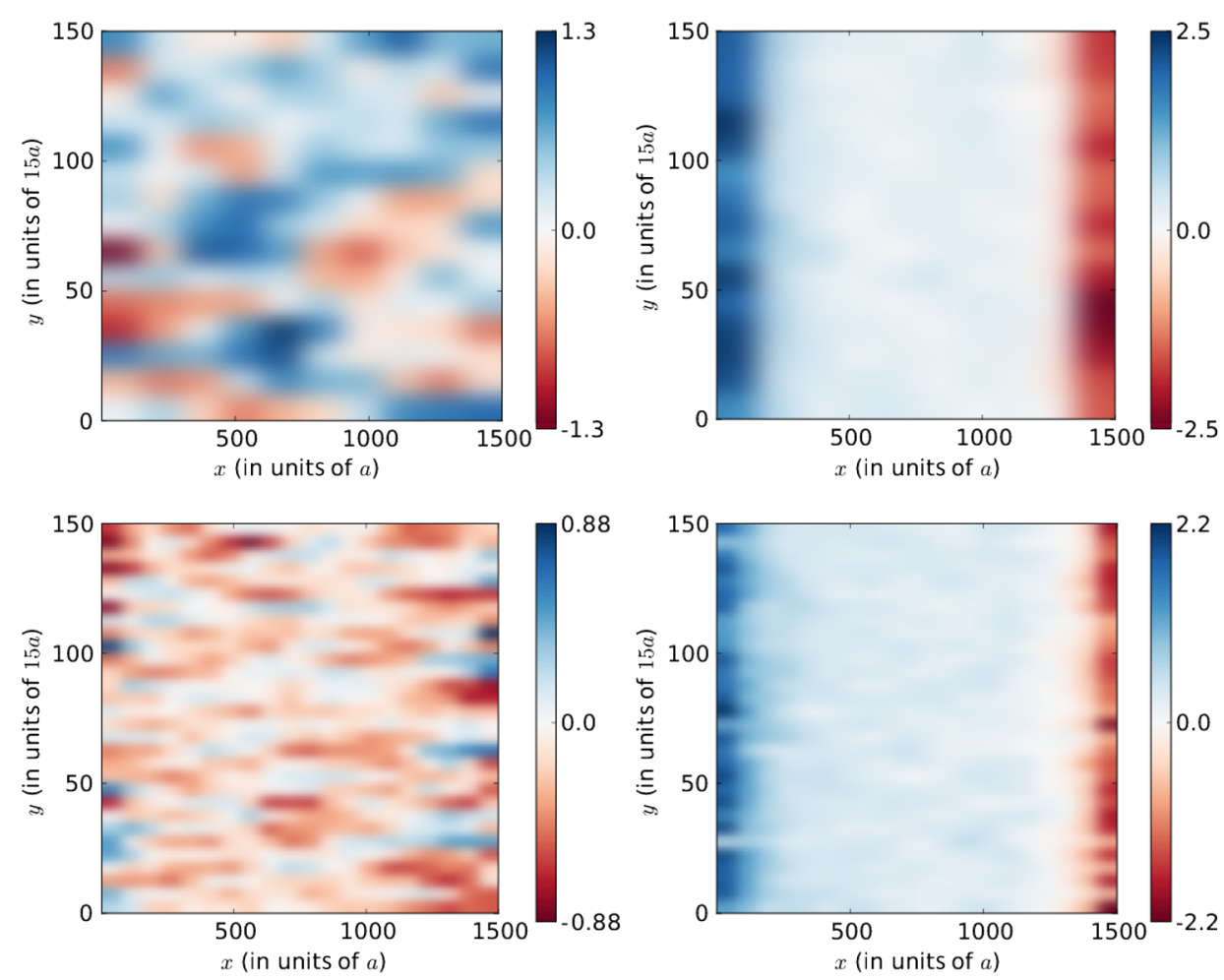

FIG. 4. Map of the local heat exchanges $\mathcal{I}_{x, y}^{Q}$ between the NWs and the phonon bath (substrate), in units of $10^{-3} t^{2} / \hbar$, at the band center $\left(V_{g}=0\right.$, left) and near the lower band edge $\left(V_{g}=2.25 t\right.$, right), for $k_{B} T=0.25 t$ (top) and $k_{B} T=0.5 t$ (bottom). When phonons are absorbed by NW charge carriers in the small area of size $\Lambda_{\mathrm{ph}}^{2}$ around $(x, y), \mathcal{I}_{x, y}^{Q}>0$ and the substrate below is locally cooled down (blue). When phonons are released, $\mathcal{I}_{x, y}^{Q}<0$ and the substrate is locally heated up (red). As explained in the text, we took $\Lambda_{\mathrm{ph}}=75 a$ for $k_{B} T=0.5 t$ and $\Lambda_{\mathrm{ph}}=150 a$ for $k_{B} T=0.25 t$. Note that the formation of hot and cold spots at the boundaries of the NWs is clearly visible for both temperatures when $V_{g}$ is tuned in order to probe their band edges (right), while no net effect is evident in the absence of any gate voltage (left). In all panels, data have been plotted for $M=150 \mathrm{NWs}$ of length $L=1500 a$ with interspacing $15 a$. Other parameters are $W=t, \gamma_{e}=\gamma_{e-\mathrm{ph}}=t / \hbar$, and $\delta \mu=10^{-3} t$.

maximal distance $L_{M}$ in space or $\Delta$ in energy [67]) only above $\mu$. Therefore, they need to absorb phonons to reach higher-energy states (blue region). After a few hops, having climbed at higher energies, they continue propagating with equal probabilities of having upward or downward energy hops (white region). On reaching the other end, they progressively climb down, i.e., release heat to the substrate (red region), until they reach $\mu$ and tunnel out into the right reservoir. As a consequence, the substrate regions below the NW extremities are cooled on the source side and heated on the drain side [see Fig. 1(b)]. A comparison between the top and bottom panels of Fig. 4 shows us that the heat maps are not much modified when the temperature is doubled [from $k_{B} T=0.25 t$ (top) to $k_{B} T=0.5 t$ (bottom)]. The fact that the surface $\Lambda_{\mathrm{ph}} \times \Lambda_{\mathrm{ph}}$ inside which the heat currents are summed up is smaller at larger temperature $\left(\Lambda_{\mathrm{ph}}=75 a\right.$ at $k_{B} T=0.5 t$ instead of $\Lambda_{\mathrm{ph}}=150 a$ at $\left.k_{B} T=0.25 t\right)$ is compensated by a smoothing of the $I_{i}^{Q}$ fluctuations. This makes the hot and cold strips still clearly visible and well defined in the bottom right panel of Fig. 4.

We point out that the maximum values of $\mathcal{I}_{x, y}^{Q}$ are roughly of the same order of magnitude with or without the gate (see the scale bars in Fig. 4). The advantage of using a gate is the ability to split the positive and negative $\mathcal{I}_{x, y}^{Q}$ regions into two well-separated spots at the NW extremities. One can then imagine to exploit the cold spot in the substrate to cool down a hot spot of an electronic circuit put in close proximity. Let us also stress that the assumption of elastic tunneling processes between the electrodes and the NWs is not necessary to observe the gate-induced hot and cold spots. The latter arise from the "climbing" up or down in energy that charge carriers, at $\mu$ far into the electrodes, must undergo in order to hop through the NWs (hopping transport being favored around the impurity band center in the NWs). Though in our model heat exchanges take place only inside the NWs, phonon emission (absorption) will actually take place also at the electrode extremities, roughly within an inelastic relaxation length from the contacts. This has clearly no qualitative impact, as it amounts to only a slight shift or smearing of the hot and cold spots.

Finally, let us estimate the cooling powers associated to the data shown in Fig. 4. Assuming again $t / k_{B} \approx 150 \mathrm{~K}$ and $a \approx 3.2 \mathrm{~nm}$, we find that a value of $\mathcal{I}_{x, y}^{Q}=10^{-3}\left(t^{2} / \hbar\right)$ in Fig. 4 (bottom) corresponds to a cooling power density of the order of $8 \times 10^{-10} \mathrm{~W} \mu \mathrm{m}^{-2}$ at the temperature 
considered $T=0.5 t / k_{B} \approx 77 \mathrm{~K}$ (the boiling temperature of liquid nitrogen at atmospheric pressure), for which $\Lambda_{\mathrm{ph}} \approx$ $240 \mathrm{~nm}$ in $\mathrm{SiO}_{2}$. We underline that this order of magnitude is obtained for a given set of parameters, in particular, for an infinitesimal bias $\delta \mu=10^{-3} t \approx 13 \mu \mathrm{V}$ that guarantees to remain in the linear response regime. It should not be taken in the strict sense but only as a benchmark value to fix ideas. For instance, according to this estimation, one should be able in principle to reach cooling power densities $\approx 6 \times$ $10^{-8} \mathrm{~W} \mu \mathrm{m}^{-2}$ by applying a larger bias $\delta \mu / e \approx 1 \mathrm{mV}$. To be more specific, we note that the geometry considered in Fig. 4 is realized [68] with a bidimensional array of 150, 5- $\mu \mathrm{m}$-long NWs, covered by two 7.2- $\mu \mathrm{m}$-long (or longer) metallic electrodes. For this geometry and at $T \approx 77 \mathrm{~K}$, the areas of the cooled and heated regions are approximately $7.2 \times 0.25 \approx 2 \mu \mathrm{m}^{2}$ (see the lower right panel of Fig. 4), but if one considered $1-\mathrm{cm}$ electrodes covering $2 \times 10^{5} \mathrm{NWs}$, those areas would naturally extend. Thus, for a bias $\delta \mu / e \approx 1 \mathrm{mV}$ and a temperature $T \approx 77 \mathrm{~K}$, our setup would allow one to take approximately $0.15 \mathrm{~mW}$ in a strip of $1 \mathrm{~cm} \times 0.25 \mu \mathrm{m}$ area and $0.25-\mu \mathrm{m}$ thickness located in the $\mathrm{SiO}_{2}$ substrate below the source electrode and to transfer it in another strip of similar size located at $5 \mu \mathrm{m}$ away below the drain electrode. Obviously, the longer the NWs, the longer would be the scale of the heat transfer. The larger the bias and the number of used NWs, the larger would be the heat transfer.

\section{CONCLUSION}

The low carrier density of a doped semiconductor can be varied by applying a voltage on a (back, side, or front) metallic gate. This led us to study thin and weakly doped semiconductor NWs, where electron transport is activated, instead of thick metallic NWs (with much larger electrical and thermal conductances) where the field effects are negligible. Considering arrays of these NWs in the FET configuration, we have focused our attention on the activated regime which characterizes a very broad temperature domain in amorphous semiconductors [57]. When charge transport between localized states is thermally assisted by phonons, we have shown that the absorption or the emission of phonons in strips located near the source and drain electrodes can be controlled with a back gate. This opens new perspectives for managing heat at submicron scales. By tuning the electrochemical potential $\mu$ near the band edges of the NW impurity band, we have studied how to take advantage of electron-phonon coupling for energy harvesting and hot-spot cooling. Our estimates indicate that large power factors are reachable in these arrays, with good thermoelectric figures of merit.

\section{ACKNOWLEDGMENTS}

This work was supported by CEA within the DSMEnergy Program (Project No. E112-7-Meso-Therm-DSM).
We thank O. Bourgeois, Y. Imry, and F. Ladieu for stimulating discussions.

Note added in proof.-K. A. Muttalib and S. Hershfield recently published a paper (Ref. [73]) which also deals with thermoelectric transport in gated disordered nanowires. Considering a nanowire array working as an energy harvester in the nonlinear response regime, the authors study how to optimize the efficiency and the electric output power of the device by tuning the external gate voltage. Their results are derived in the low-temperature coherenttransport regime, and not in the activated inelastic regime which we consider.

\section{APPENDIX A: RESOLUTION OF THE RANDOM- RESISTOR-NETWORK PROBLEM}

Hereafter, we summarize the numerical method used to solve the random-resistor-network problem [46,50,57]. The three-terminal setup configuration is recalled in Fig. 5, with emphasis on the hopping transport mechanism taking place in the NWs. Starting from a set of states $i$ localized at positions $x_{i}$ inside the NWs, with energies $E_{i}$ and localization lengths $\xi_{i}$, we first evaluate the transition rates $\Gamma_{i \alpha}$ from the localized state $i$ to the reservoir $\alpha=L$ or $R$ and $\Gamma_{i j}$ from states $i$ to $j$ within the same wire (interwire hopping being neglected). They are given by the Fermi golden rule as

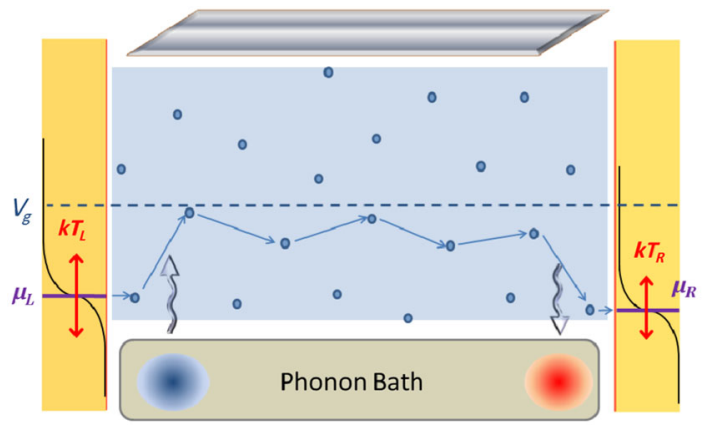

FIG. 5. Phonon-assisted hopping transport through the localized states (dots) of a disordered NW connected to two electrodes $L$ and $R$ and to a phonon bath. The electronic reservoirs $L$ and $R$ are thermalized at temperatures $T_{L[R]}$ and held at electrochemical potentials $\mu_{L[R]}$ (their Fermi functions are sketched by the black curves on both sides). A metallic gate (shaded gray plate drawn on top) allows one to shift the NW impurity band (blue central region). Here, the gate potential $V_{g}$ is adjusted such that electrons tunnel in and out of the electronic reservoirs near the lower edge of the impurity band. Therefore, electrons tend to absorb phonons at the entrance in order to reach available states of higher energies and to emit phonons on the way out. The two wavy arrows indicate the local heat flows between the NW electrons and the phonon bath. They give rise to a pair of cold (blue) and hot (red) spots in the substrate beneath the NW (in the deposited setup configuration). 


$$
\begin{gathered}
\Gamma_{i \alpha}=\gamma_{i \alpha} f_{i}\left[1-f_{\alpha}\left(E_{i}\right)\right], \\
\Gamma_{i j}=\gamma_{i j} f_{i}\left(1-f_{j}\right)\left[N_{i j}+\theta\left(E_{i}-E_{j}\right)\right],
\end{gathered}
$$

where $f_{i}$ is the occupation probability of state $i, f_{\alpha}(E)=$ $\left\{\exp \left[\left(E-\mu_{\alpha}\right) / k_{B} T_{\alpha}\right]+1\right\}^{-1}$ is the Fermi distribution of reservoir $\alpha, N_{i j}=\left[\exp \left(\left|E_{j}-E_{i}\right| / k_{B} T\right)-1\right]^{-1}$ is the probability of having a phonon with energy $\left|E_{j}-E_{i}\right|$ assisting the hop, and $\theta$ is the Heaviside function. In Eq. (A1), $\gamma_{i \alpha}=\gamma_{e} \exp \left(-2 x_{i \alpha} / \xi_{i}\right), x_{i \alpha}$ denoting the distance of state $i$ from reservoir $\alpha$ and $\gamma_{e}$ being a constant quantifying the coupling from the localized states in the NW to the extended states in the reservoirs. Usually, $\xi_{i} \approx \xi(\mu)$ is assumed and the rate $\gamma_{i j}$ in Eq. (A2) is simply given by $\gamma_{i j}=\gamma_{e-\mathrm{ph}} \exp \left[-2 x_{i j} / \xi(\mu)\right]$, with $x_{i j}=\left|x_{i}-x_{j}\right|$ and $\gamma_{e-\mathrm{ph}}$ measuring the electron-phonon coupling. Since this approximation does not hold in the vicinity of the impurity band edges, where the localization lengths vary strongly with the energy, we use a generalized expression for $\gamma_{i j}$ that accounts for the different localization lengths $\xi_{i} \neq \xi_{j}$ (see Ref. [40]).

By using Eqs. (A1) and (A2) and imposing charge conservation at each network node $i$, we deduce the $N f_{i}$ 's of the $M$ independent NWs. The charge and heat currents flowing from reservoir $\alpha$ to the system can then be calculated as $I_{\alpha}^{e}=e \sum_{i} I_{\alpha i}$ and $I_{\alpha}^{Q}=\sum_{i} I_{\alpha i}\left(E_{i}-\mu_{\alpha}\right)$, where $I_{\alpha i}=\Gamma_{\alpha i}-\Gamma_{i \alpha}$ and $e$ is the electron charge. In principle, the heat current $I_{P}^{Q}=(1 / 2) \sum_{i} I_{i}^{Q}$ coming from the phonon bath can be calculated as well, but in this work, we investigate only the behavior of the local heat currents $I_{i}^{Q}=\sum_{j}\left(E_{j}-E_{i}\right) I_{i j}^{N}$ with $I_{i j}^{N}=\Gamma_{i j}-\Gamma_{j i}$. Without loss of generality, we choose the right terminal $R$ as the reference; i.e., we set $\mu_{R}=\mu, T_{R}=T$ and we impose on the left side $\mu_{L}=\mu+\delta \mu, T_{L}=T+\delta T$. Using the Onsager formalism, we relate the particle $\left(I_{L}^{e}\right)$ and heat $\left(I_{L}^{Q}\right)$ currents computed in linear response to the small imposed bias $\delta \mu$ and $\delta T$ [58]. This allows us to deduce the thermoelectric coefficients $G$, $K^{e}$, and $S$.

\section{APPENDIX B: SIZE EFFECTS}

We investigate the effects on the various transport coefficients $G, K^{e}$, and $S$, the power factor $\mathcal{Q}$, and the electronic figure of merit $Z_{e} T$ of varying the length $L$ of the NWs. The results are shown in Fig. 6, for three values of the temperatures $k_{B} T=0.1 t, 0.5 t$, and $1.0 t$ and for two configurations corresponding to bulk $\left(V_{g}=t\right)$ and edge transport $\left(V_{g}=2.5 t\right)$. In all cases (except the one for $k_{B} T=0.1 t$ and $V_{g}=2.5 t$, solid red circles in Fig. 6), electronic transport through the NWs is thermally activated (see Fig. 3 in Ref. [40]), and the results are seen to be essentially size independent, as expected in the activated regime. In the case identified by solid red circles in Fig. 6 and corresponding to the lowest temperature and the vicinity of the band edge, transport turns out to be achieved
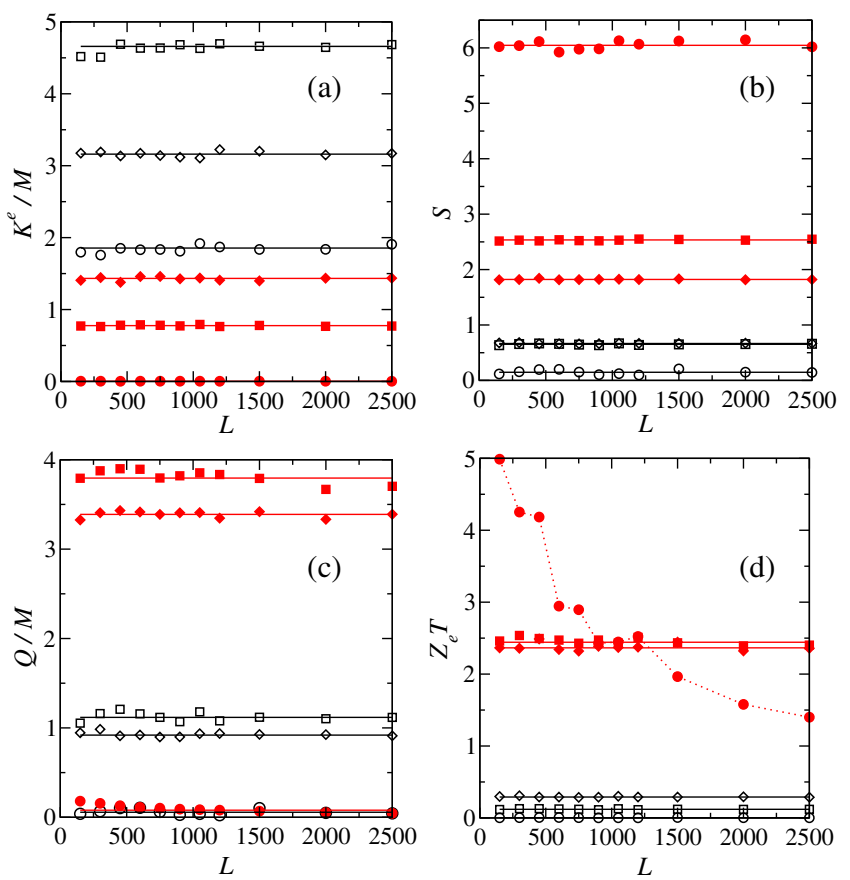

FIG. 6. Behavior of the transport coefficients as a function of the NW length $L$ (in units of the spacing $a$ ). Panels show (a) the rescaled electronic contribution to the thermal conductance $K^{e}$ (in units of $k_{B} t / \hbar$ ), (b) the thermopower $S$ (in units of $k_{B} / e$ ), (c) the rescaled power factor $\mathcal{Q}$ (in units of $k_{B}^{2} / \hbar$ ), and (d) the electronic figure of merit $Z_{e} T$. In all four panels, data are plotted for $k_{B} T=$ $0.1 t$ (circles), $k_{B} T=0.5 t$ (squares), and $k_{B} T=t$ (rhombus), in the case of bulk transport $\left(V_{g}=t\right.$, empty symbols) and edge transport ( $V_{g}=2.5 t$, full symbols). Lines are guides to the eye. Other parameters are fixed to $W=t$ and $\gamma_{e}=\gamma_{e-\mathrm{ph}}=t / \hbar$.

by elastic tunneling processes: The electrical conductance becomes size dependent, which causes the electronic figure of merit $Z_{e} T$ to decrease roughly as $1 / L$. However, being interested in the activated regime and, in particular, in the regime of temperatures where the power factor is largest $\left(k_{B} T \simeq 0.5 t\right)$, we can conclude that the size effects on the results shown in this work are completely negligible. Also, we note that the small fluctuations observed especially at the smallest sizes in Fig. 6 are a consequence of having taken a finite number of parallel NWs $(M=150)$ : They would diminish in the limit $M \rightarrow \infty$ due to self-averaging.

\section{APPENDIX C: ON THE DEPENDENCE ON THE COUPLINGS $\gamma_{e}$ AND $\gamma_{e-\mathrm{ph}}$}

In this Appendix, we investigate how the transport coefficients $G, K^{e}$, and $S$, the power factor $\mathcal{Q}=S^{2} G$, and the electronic figure of merit $Z_{e} T=S^{2} G T / K^{e}$ are modified upon varying the couplings $\gamma_{e}$ and $\gamma_{e \text {-ph }}$ of the localized states with the electrodes and the phonon bath, respectively. We introduce the notation $\alpha \equiv \gamma_{e-\mathrm{ph}} / \gamma_{e}$. We first notice that if $\alpha$ is kept fixed, the electrical conductance $G$ and the electronic thermal conductance $K^{e}$ are strictly proportional to $\gamma_{e}$, while the thermopower $S$ is independent 

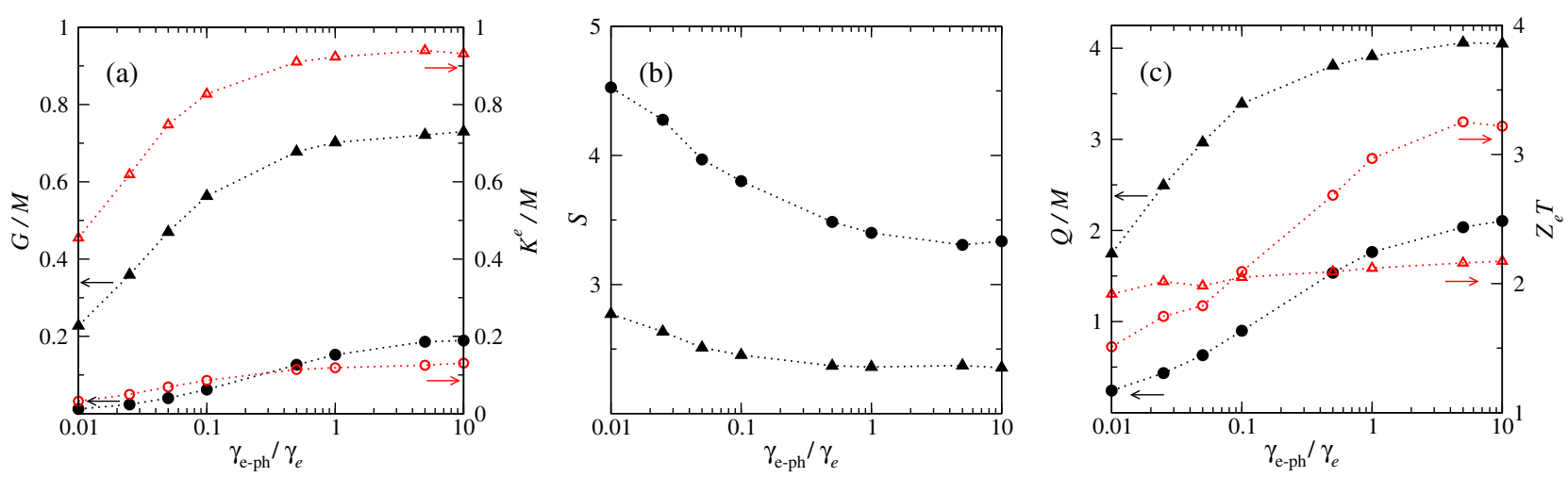

FIG. 7. Dependency of $G, K^{e}, S, \mathcal{Q}$, and $Z_{e} T$ on the ratio $\gamma_{e \text {-ph }} / \gamma_{e}$. (a) Electrical $\left(G / M\right.$, black full symbols) and thermal $\left(K^{e} / M\right.$, red empty symbols) conductances, in units of $e^{2} / \hbar$ and $k_{B} t / \hbar$, respectively. (b) Thermopower in units of $k_{B} / e$. (c) $\mathcal{Q} / M$ in units of $k_{B}^{2} / \hbar$ (black full symbols) and $Z_{e} T$ (red empty symbols). In all panels, different symbols correspond to $k_{B} T=0.2 t$ (circles) and $k_{B} T=0.5 t$ (triangles), while dotted lines are guides to the eye. Data have been plotted for a given set of $M=150$ parallel NWs of length $L=450 a$, with $\gamma_{e}=t / \hbar, W=t$, and $V_{g}=2.4 t$. Note that when $\gamma_{e \text {-ph }} \gtrsim \gamma_{e}$ all these coefficients are nearly constant.

of it. This behavior is a direct consequence of the formulation of the random-resistor-network problem and can be seen at the stage of writing the equations (see Ref. [40]), before solving them numerically. Therefore, for any fixed $\alpha$, $\mathcal{Q} / \gamma_{e}$ and $Z_{e} T$ are necessarily independent of the choice of $\gamma_{e}$. We thus find that $G / \gamma_{e}, K^{e} / \gamma_{e}, S, \mathcal{Q} / \gamma_{e}$, and $Z_{e} T$ are functions of the single parameter $\alpha$ and not of the couple of parameters $\gamma_{e}$ and $\gamma_{e-\mathrm{ph}}$ separately. Those functions are plotted in Fig. 7 for two different temperatures. The conductances, the power factor, and the figure of merit increase with $\alpha$ (as long as lack of phonons is a limiting factor to transport through the NWs), while the thermopower decreases. All of them tend to saturate for $\alpha \gtrsim 1$. This shows us, inter alia, that $\mathcal{Q} / \gamma_{e}$ and $Z_{e} T$ are essentially independent of $\gamma_{e}$ and $\gamma_{e \text {-ph }}$ if $\gamma_{e \text {-ph }} \gtrsim \gamma_{e}$ and that they only deviate slowly from this limit if $\gamma_{e-\mathrm{ph}}<\gamma_{e}$. Such a robustness of $\mathcal{Q} / \gamma_{e}$ and $Z_{e} T$ to variations of $\gamma_{e}$ and $\gamma_{e \text {-ph }}$ reinforces the impact of the results shown in this work.

\section{APPENDIX D: ESTIMATION OF THE PHONON THERMALIZATION LENGTH}

We show in Fig. 8 an example of the map of the raw heat currents $I_{i}^{Q}$ locally exchanged between the NWs and the substrate [see Eq. (2)]. We see that the $I_{i}^{Q}$, s fluctuate between positive and negative values at random positions of the substrate and that no net effect emerges. As discussed in Sec. V, the formation of the hot and cold spots is a process which becomes visible only upon summing in a single term $\mathcal{I}_{x, y}^{Q}$ all the contributions $I_{i}^{Q}$ coming from states $i$ located within an area $\Lambda_{\mathrm{ph}} \times \Lambda_{\mathrm{ph}}$ around the point of coordinates $(x, y) . \Lambda_{\mathrm{ph}}$, which represents the thermalization length of the substrate, is given by the inelastic phonon mean free path: This quantity may be different for different phonon wavelengths, and, while it does not change much around room temperature, it can vary significantly at lower temperatures. It is possible to relate $\Lambda_{\mathrm{ph}}$ to the dominant phonon wavelength [69] as $\Lambda_{\mathrm{ph}}=300 \lambda_{\mathrm{ph}}^{\text {dom }}$, where the coefficient 300 is for $\mathrm{SiO}_{2}$ and may be different for other materials. This allows the calculation of the thermalization length $\Lambda_{\mathrm{ph}}$, once $\lambda_{\mathrm{ph}}^{\text {dom }}$ is known. According to Refs. [70,71], the latter can be estimated as

$$
\lambda_{\mathrm{ph}}^{\mathrm{dom}} \simeq \frac{h v_{s}}{4.25 k_{B} T},
$$

where $h$ is the Planck constant. Taking $v_{s}=5300 \mathrm{~m} / \mathrm{s}$ as the sound velocity in $\mathrm{SiO}_{2}$ [71], we can easily deduce $\lambda_{\mathrm{ph}}^{\mathrm{dom}} \simeq 0.2 \mathrm{~nm}$ from which $\Lambda_{\mathrm{ph}} \simeq 60 \mathrm{~nm}$ at room temperature $T=300 \mathrm{~K}$. Values of $\Lambda_{\mathrm{ph}}$ at other (not vanishing) temperatures follow immediately from the temperature dependence in Eq. (D1). We shall stress that the real values of $\Lambda_{\text {ph }}$ may differ from our prediction by a small

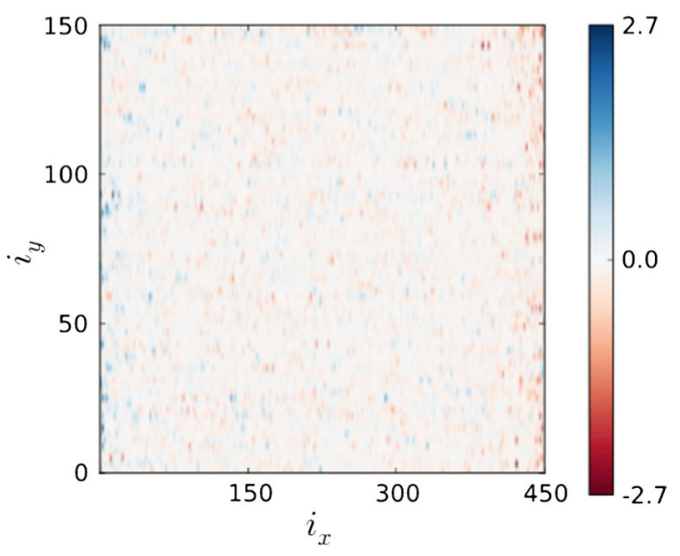

FIG. 8. Map of the local heat currents $I_{i}^{Q}$ exchanged between the NWs and the substrate at each NWs site $i=\left(i_{x}, i_{y}\right)$, in units of $10^{-7} t^{2} / \hbar$, for $k_{B} T=0.05 t$ and $V_{g}=2.25 t$. The horizontal coordinate is the position along the NWs, while the vertical one labels each NW. The presence of hot and cold spots is hidden by the fluctuations. They emerge when the raw $I_{i}^{Q}$ data are summed up within areas of size $\Lambda_{\mathrm{ph}} \times \Lambda_{\mathrm{ph}}$. Parameters: $M=150$, $L=450 a, W=t, \gamma_{e}=\gamma_{e-\mathrm{ph}}=t / \hbar$, and $\delta \mu=10^{-5} t$. 
numerical factor, which however is not important within our qualitative approach. To convert these lengths in the units used in Sec. V, we assume the average distance between localized states $a \approx 3.2 \mathrm{~nm}$ in highly doped silicon NWs, which together with $t / k_{B} \approx 150 \mathrm{~K}$ allows us to estimate, for example, $\Lambda_{\mathrm{ph}} \approx 75 a$ at $T=0.5 t / k_{B}=75 \mathrm{~K}$.

[1] L. D. Hicks and M. S. Dresselhaus, Thermoelectric figure of merit of a one-dimensional conductor, Phys. Rev. B 47, 16631 (1993).

[2] B. M. Curtin, E. W. Fang, and J. E. Bowers, Highly ordered vertical silicon nanowire array composite thin films for thermoelectric devices, J. Electron. Mater. 41, 887 (2012).

[3] C. Blanc, A. Rajapbour, S. Volz, T. Fournier, and O. Bourgeois, Phonon heat conduction in corrugated silicon nanowires below the Casimir limit, Appl. Phys. Lett. 103, 043109 (2013).

[4] Y. M. Brovman, J. P. Small, Y. Hu, Y. Fang, C. M. Lieber, and P. Kim, Electric field effect thermoelectric transport in individual silicon and germanium/silicon nanowire, arXiv: 1307.0249 .

[5] A. Stranz, A. Waag, and E. Peiner, High-temperature performance of stacked silicon nanowires for thermoelectric power generation, J. Electron. Mater. 42, 2233 (2013).

[6] S. Karg, P. Mensch, B. Gotsmann, H. Schmid, P. Das Kanungo, H. Ghoneim, V. Schmidt, M. T. Björk, V. Troncale, and H. Riel, Measurement of thermoelectric properties of single semiconductor nanowires, J. Electron. Mater. 42, 2409 (2013).

[7] S. Roddaro, D. Ercolani, M. A. Safeen, S. Suomalainen, F. Rossella, F. Giazotto, L. Sorba, and F. Beltram, Giant thermovoltage in single InAs nanowire field-effect transistors, Nano Lett. 13, 3638 (2013).

[8] J. Moon, J.-H. Kim, Z. C. Y. Chen, J. Xiang, and R. Chen, Gate-modulated thermoelectric power factor of hole gas in GeSi coreshell nanowires, Nano Lett. 13, 1196 (2013).

[9] Y. Tian, M. R. Sakr, J. M. Kinder, D. Liang, M. J. MacDonald, R. L. J. Qiu, H.-J. Gao, and X. P. A. Gao, One-dimensional quantum confinement effect modulated thermoelectric properties in InAs nanowires, Nano Lett. 12, 6492 (2012).

[10] A. I. Hochbaum, R. Chen, R. D. Delgado, W. Liang, E. C. Garnett, M. Najarian, A. Majumdar, and P. Yang, Enhanced thermoelectric performance of rough silicon nanowires, Nature (London) 451, 163 (2008).

[11] M. Z. Atashbar, D. Banerji, S. Singamaneni, and V. Bliznyuk, Deposition of parallel arrays of palladium nanowires and electrical characterization using microelectrode contacts, Nanotechnology 15, 374 (2004).

[12] R. Yerushalmi, Z. A. Jacobson, J. C. Ho, Z. Fan, and A. Javey, Large scale, highly ordered assembly of nanowire parallel arrays by differential roll printing, Appl. Phys. Lett. 91, 203104 (2007).

[13] M. C. P. Wang and B. D. Gates, Directed assembly of nanowires, Mater. Today 12, 34 (2009).

[14] G. Zhang, K. Tateno, H. Gotoh, and H. Nakano, Parallelaligned GaAs nanowires with $\langle 110\rangle$ orientation laterally grown on [311]B substrates via the gold-catalyzed vapor-liquid-solid mode, Nanotechnology 21, 095607 (2010).

[15] D. Davila, A. Tarancon, M. Fernandez-Regulez, C. Calaza, M. Salleras, A. San Paulo, and L. Fonseca, Silicon nanowire arrays as thermoelectric material for a power microgenerator, J. Micromech. Microeng. 21, 104007 (2011).

[16] R. A. Farrell, N. T. Kinahan, S. Hansel, K. O. Stuen, N. Petkov, M. T. Shaw, L. E. West, V. Djara, R. J. Dunne, O. G. Varona, P. G. Gleeson, Jung S.-J, H.-Y. Kim, M. M. Koleśnik, T. Lutz, C. P. Murray, J. D. Holmes, P. F. Nealey, G. S. Duesberg, V. Krstić, and M. A. Morris, Large-scale parallel arrays of silicon nanowires via block copolymer directed self-assembly, Nanoscale 4, 3228 (2012).

[17] S. Pregl, W. M. Weber, D. Nozaki, J. Kunstmann, L. Baraban, J. Opitz, T. Mikolajick, and G. Cuniberti, Parallel arrays of Schottky barrier nanowire field effect transistors: Nanoscopic effects for macroscopic current output, Nano Res. 6, 381 (2013).

[18] A. I. Persson, L. E. Fröberg, L. Samuelson, and H. Linke, The fabrication of dense and uniform InAs nanowire arrays, Nanotechnology 20, 225304 (2009).

[19] A. R. Abramson, W. C. Kim, S. T. Huxtable, H. Yan, Y. Wu, A. Majumdar, C.-L. Tien, and P. Yang, Fabrication and characterization of a nanowire/polymer-based nanocomposite for a prototype thermoelectric device, Microelectromech. Syst. 13, 505 (2004).

[20] J. Keyani, A. M. Stacy, and J. Sharp, Assembly and measurement of a hybrid nanowire-bulk thermoelectric device, Appl. Phys. Lett. 89, 233106 (2006).

[21] M. F. O’Dwyer, T. E. Humphrey, and H. Linke, Concept study for a high-efficiency nanowire based thermoelectric, Nanotechnology 17, S338 (2006).

[22] T. Markussen, A.-P. Jauho, and M. Brandbyge, Electron and phonon transport in silicon nanowires: Atomistic approach to thermoelectric properties, Phys. Rev. B 79, 035415 (2009).

[23] G. Liang, W. Huang, C. S. Koong, J.-S. Wang, and J. Lan, Geometry effects on thermoelectric properties of silicon nanowires based on electronic band structures, J. Appl. Phys. 107, 014317 (2010).

[24] G. Gumbs, A. Balassis, and D. Huang, Energy bands, conductance, and thermoelectric power for ballistic electrons in a nanowire with spin-orbit interaction, J. Appl. Phys. 108, 093704 (2010).

[25] N. Neophytou, M. Wagner, H. Kosina, and S. Selberherr, Analysis of thermoelectric properties of scaled silicon nanowires using an atomistic tight-binding model, J. Electron. Mater. 39, 1902 (2010).

[26] B. Wang, J. Zhou, R. Yang, and B. Li, Ballistic thermoelectric transport in structured nanowires, New J. Phys. 16, 065018 (2014).

[27] Y.-M. Lin, X. Sun, and M. S. Dresselhaus, Theoretical investigation of thermoelectric transport properties of cylindrical Bi nanowires, Phys. Rev. B 62, 4610 (2000).

[28] T. E. Humphrey and H. Linke, Reversible Thermoelectrics Nanomaterials, Phys. Rev. Lett. 94, 096601 (2005).

[29] I. Bejenari and V. Kantser, Thermoelectric properties of bismuth telluride nanowires in the constant relaxation-time approximation, Phys. Rev. B 78, 115322 (2008). 
[30] T. T. M. Vo, A. J. Williamson, V. Lordi, and G. Galli, Atomistic design of thermoelectric properties of silicon nanowires, Nano Lett. 8, 1111 (2008).

[31] L. Shi, D. Yao, G. Zhang, and B. Li, Size dependent thermoelectric properties of silicon nanowires, Appl. Phys. Lett. 95, 063102 (2009).

[32] I. Bejenari, V. Kantser, and A. A. Balandin, Thermoelectric properties of electrically gated bismuth telluride nanowires, Phys. Rev. B 81, 075316 (2010).

[33] N. Neophytou and H. Kosina, Numerical study of the thermoelectric power factor in ultra-thin $\mathrm{Si}$ nanowires, J. Comput. Electron. 11, 29 (2012).

[34] E. B. Ramayya, L. N. Maurer, A. H. Davoody, and I. Knezevic, Thermoelectric properties of ultrathin silicon nanowires, Phys. Rev. B 86, 115328 (2012).

[35] N. Neophytou and H. Kosina, Gated Si nanowires for large thermoelectric power factors, Appl. Phys. Lett. 105, 073119 (2014).

[36] I. Bejenari and P. Kratzer, Atomistic calculation of the thermoelectric properties of Si nanowires, Phys. Rev. B 90, 045429 (2014).

[37] B. M. Curtin and J. E. Bowers, Thermoelectric power factor enhancement with gate-all-around silicon nanowires, J. Appl. Phys. 115, 143704 (2014).

[38] A. H. Davoody, E. B. Ramayya, L. N. Maurer, and I. Knezevic, Ultrathin GaN nanowires: Electronic, thermal, and thermoelectric properties, Phys. Rev. B 89, 115313 (2014).

[39] R. Bosisio, G. Fleury, and J.-L. Pichard, Gate-modulated thermoelectric conversion of 1-D disordered nanowires: I. Low temperature coherent regime, New J. Phys. 16, 035004 (2014).

[40] R. Bosisio, C. Gorini, G. Fleury, and J.-L. Pichard, Gatemodulated thermoelectric conversion of 1-D disordered nanowires: II. Variable-range hopping regime, New J. Phys. 16, 095005 (2014).

[41] I. P. Zvyagin, On the theory of hopping transport in disordered semiconductors, Phys. Status Solidi (b) 58, 443 (1973).

[42] I. P. Zvyagin, in Hopping Transport in Solids, edited by M. Pollak and B. I. Shklovskii (North-Holland, Amsterdam, 1991).

[43] B. Movaghar and W. Schirmacher, On the theory of hopping conductivity in disordered systems, J. Phys. C 14, 859 (1981).

[44] K. I. Wysokinski and W. Brenig, On thermopower in hopping transport, Z. Phys. B 59, 127 (1985).

[45] J-H. Jiang, O. Entin-Wohlman, and Y. Imry, Thermoelectric three-terminal hopping transport through one-dimensional nanosystems, Phys. Rev. B 85, 075412 (2012).

[46] J-H. Jiang, O. Entin-Wohlman, and Y. Imry, Hopping thermoelectric transport in finite systems: Boundary effects, Phys. Rev. B 87, 205420 (2013).

[47] G. D. Mahan, Figure of merit for thermoelectrics, J. Appl. Phys. 65, 1578 (1989).

[48] A. Shakouri, Recent developments in semiconductor thermoelectric physics and materials, Annu. Rev. Mater. Res. 41, 399 (2011).

[49] B. Shklovskii and A. Efros, Electronic Properties of Doped Semiconductors (Springer-Verlag, Berlin, 1984).

[50] A. Miller and E. Abrahams, Impurity conduction at low concentrations, Phys. Rev. 120, 745 (1960).
[51] A. Vassighi and M. Sachdev, Thermal and Power Management of Integrated Circuits (Springer, New York, 2006).

[52] J. P. Pekola and F. W. J. Hekking, Normal-MetalSuperconductor Tunnel Junction as a Brownian Refrigerator, Phys. Rev. Lett. 98, 210604 (2007).

[53] B. Rutten, M. Esposito, and B. Cleuren, Reaching optimal efficiencies using nanosized photoelectric devices, Phys. Rev. B 80, 235122 (2009).

[54] A. Levy and R. Kosloff, Quantum Absorption Refrigerator, Phys. Rev. Lett. 108, 070604 (2012).

[55] A. Mari and J. Eisert, Cooling by Heating: Very Hot Thermal Light Can Significantly Cool Quantum Systems, Phys. Rev. Lett. 108, 120602 (2012).

[56] B. Cleuren, B. Rutten, and C. Van den Broeck, Cooling by Heating: Refrigeration Powered by Photons, Phys. Rev. Lett. 108, 120603 (2012).

[57] V. Ambegaokar, B. I. Halperin, and J. S. Langer, Hopping conductivity in disordered systems, Phys. Rev. B 4, 2612 (1971).

[58] H. B. Callen, Thermodynamics and an Introduction to Thermostatics (Wiley, New York, 1985).

[59] We estimate $t$ by comparing the bandwidth $4 t+W$ in our model to the typical width of the impurity band in highly doped silicon NWs (see, for instance, Ref. [72]). Note that the NWs are then depleted by the field effect.

[60] G. Benenti, G. Casati, T. Prosen, and K. Saito, Fundamental aspects of steady state heat to work conversion, arXiv: 1311.4430.

[61] The use of the 1D model is justified at a semiquantitative level if the nanowire diameter is smaller than the Mott hopping length $L_{M}$ (the typical length of an electron hop along the nanowire).

[62] D. Li, Y. Wu, P. Kim, L. Shi, P. Yang, and A. Majumdar, Thermal conductivity of individual silicon nanowires, Appl. Phys. Lett. 83, 2934 (2003).

[63] M.-T. Hung, C.-C. Wang, J.-C. Hsu, J.-Y. Chiou, S.-W. Lee, T. M. Hsu, and P.-W. Li, Large reduction in thermal conductivity for $\mathrm{Ge}$ quantum dots embedded in $\mathrm{SiO} 2$ system, Appl. Phys. Lett. 101, 251913 (2012).

[64] P. Hopkins, B. Kaehr, L. M. Phinney, T. P. Koehler, A. M. Grillet, D. Dunphy, F. Garcia, and C. J. Brinker, Measuring the thermal conductivity of porous, transparent $\mathrm{SiO} 2$ films with time domain thermoreflectance, J. Heat Transfer 133, 061601 (2011).

[65] P. Scheuerpflug, M. Hauck, and J. Fricke, Thermal properties of silica aerogels between 1.4 and 330 K, J. Non-Cryst. Solids 145, 196 (1992).

[66] Practically, we map the 2D parallel NW array onto a square grid, and for each square of size $\Lambda_{\mathrm{ph}}^{2}$ we calculate the net heat current entering the NWs. For better visibility, data are then smoothed (with a standard Gaussian interpolation) to produce the heat map shown in Fig. 4 .

[67] Here $L_{M} \approx 10.6 a$ and $\Delta \approx 2.4 t$ for $k_{B} T=0.25 t$, while $L_{M} \approx 7.5 a$ and $\Delta \approx 3.3 t$ for $k_{B} T=0.5 t$.

[68] Data shown in Fig. 4 result from numerical simulations run for a set of 150 1D NWs (of length 1500a) separated from each other by a distance $15 \mathrm{a}$. They are expected to describe the physics of realistic arrays made of $150 \mathrm{NWs}$ 
covering an area of width $150 \times 15 a \approx 7.2 \mu \mathrm{m}$ and length $1500 a \approx 5 \mu \mathrm{m}$, taking again $a \approx 3.2 \mathrm{~nm}$, for instance, 150 NWs with $10-\mathrm{nm}$ diameter and $20 \%$ packing density. Other configurations could be considered as well, as long as the NW diameter is small enough for the $1 \mathrm{D}$ model to make sense and the packing density does not exceed the typical values reachable experimentally.

[69] R. O. Pohl, X. Liu, and E. Thompson, Low-temperature thermal conductivity and acoustic attenuation in amorphous solids, Rev. Mod. Phys. 74, 991 (2002).
[70] T. Klitsner and R. O. Pohl, Phonon scattering at silicon crystal surfaces, Phys. Rev. B 36, 6551 (1987).

[71] J. M. Ziman, Electrons and Phonons: The Theory of Transport Phenomena in Solids (Clarendon, New York, 1996).

[72] F. Salleh and H. Ikeda, Influence of impurity band on Seebeck coefficient in heavily-doped Si, Adv. Mater. Res. 222, 197 (2011).

[73] K. A. Muttalib and S. Hershfield, Nonlinear Thermoelectricity in Disordered Nanowires, following paper, Phys. Rev. Applied 3, 054003 (2015). 